\section{Using Projection X-ray Microscopy to Observe the Solidification Phenomena in Metals}

William F. Kaukler, The University of Alabama in Huntsville

This article outlines how projection $x$-ray microscopy can be used to view the structures that develop within solidifying metals during the freezing process (in real-time and in situ). A description of $x$-ray microscopy methods will be given. The specialized furnace design and techniques required to permit observation of solidification phenomena will also be described. This forms the outline of the salient features of our X-ray Transmission Microscope (XTM) for solidification studies. This instrument is being developed for microgravity materials research at NASA's Space Science Laborataory in the Marshall Space Flight Center, Alabama. Finally, a sampling of radiomicrographs taken during alloy solidification will be presented. By understanding how the microstructures in alloys form. we can improve the properties of materials (e.g. strength and corrosion resistance) or to design better materials.

\section{Introduction}

We can directly view, for the first time, metal solidification phenomena by (projection) $x$-ray microscopy. Only with penetrating hard $x$-rays (>10 kV) can we watch the evolution of microstructures in metals. Application of this innovative $x$-ray characterization now affords us the much needed opportunity to perform experiments with optically opaque metal alloys. This provides an unambiguous record of the three-dimensional dynamics of solidification, and thus, permits quantitative characterization of the prevailing transport conditions (convection, diffusion, phase separation and coalescence, particle pushing, etc.). The microstructure of a given metallic casting strongly determines its physical properties and. ultimately. its utility.

Past observations of this kind have been restricted to conventional optical microscopy of transparent, organic materials that behave like metals during solidification. However, unambiguous extrapolation of these results to metallic systems was impared since interfacial and transport properties of the organic model materials greatly differ from those of metals and semiconductors.

It should be made clear that this capability is right on the edge of feasibility. We are working at the intersection of the bounds of several technologies. The cliche 'pushing the envelope' comes to mind. The images
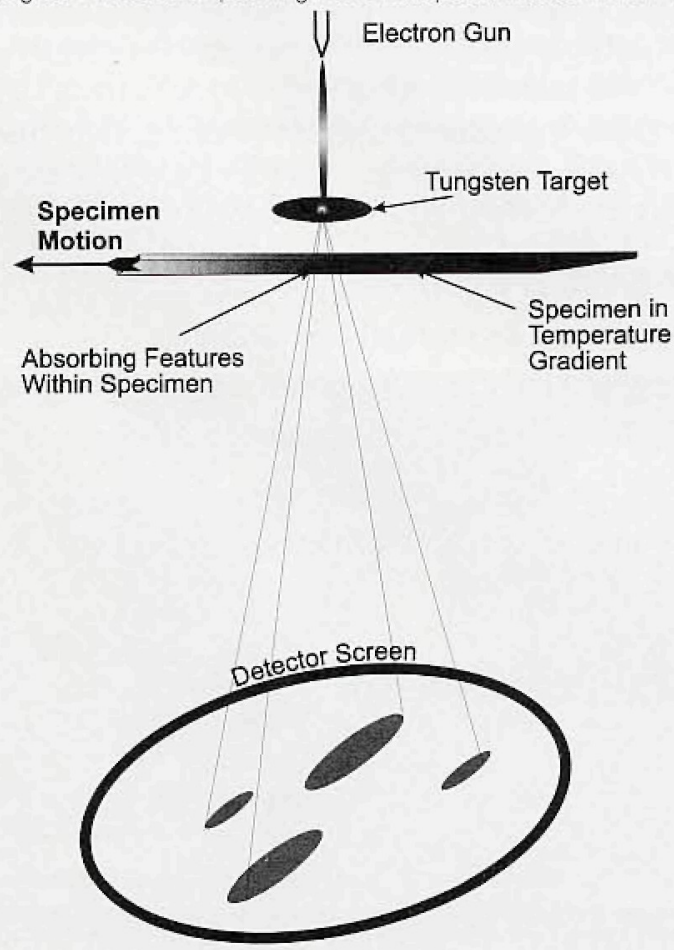

Schematic of a Projection $\mathrm{X}$-ray Microscope we have obtained to date may look unclear or fuzzy compared to the high definition images from an optical microscope or an SEM or AFM. All images require extensive processing since frequently, features have $3 \%$ or less contrast. Small, fleeting features on the monitor may never be recovered on a hard copy. Fortunately, careful alloy selection and preparation can produce some high contrast features so that even a layman can clearly see what was occurring

\section{Microscopy by Projection Radiography}

A series of limitations reduce the number of methods available to perform microscopy on metals during solidification. One is the requirement for hard $x$-rays that can penetrate metals. Another is caused by the high temperatures needed to melt metal alloys. Yet another is the need to obtain high resolutions to begin to even see the features (size range 1-100 micrometers) commonly found in solidifying alloys. Recently, the STM, AFM or related microscopies have been developed and offer atomic resolutions.... but only on a solid surface near room temperature. Solidification induced morphologies are three dimensional. If observations of exposed surfaces are made (e.g. with SEM or even polarized reflected light), the viewed microstructures will not be representative of those forming within the bulk.

The most frequent discussion of $x$-ray microscopy in the literature involves the diversity of soft $x$-ray microscopies, often using synchrotron sources or lately, pulsed laser plasmas. With these, the desire is more to break the visible light resolution barrier of conventional optics by employing the shorter wavelength of $x$-rays. Most of these methods employ scanning techniques which can only be used with stationary subjects. However the hard $x$-rays needed to see inside metals cannot be refracted to a focus by a lens. So, how does one magnify images made with hard $x$-rays? A simple approach is to pass a collimated (usually synchrotron) beam through the specimen onto photographic film which can later be optically magnified (or a photo resist that can be viewed on a SEM). Little magnification is possible and the method is not real-time. The film and specimen need to be close together (contact radiography) to keep resolutions high.

Scanning methods cannot capture the motion that solidification entails. (The interface between solid and liquid delineates the position of the conversion process from molten to crystalline metal which must travel the length of the casting.) X-ray fluorescence methods using scanned electron beams only offer near-surface feature information and usually require a vacuum that would voltalize molten metal. Grazing incidence optics, only recently beng developed. have been shown to work only in the soft $x$-ray regime although some work is underway to operate at higher photon energies like $5-10 \mathrm{keV}$. So. we are left with an old technique, little known among regular microscopists, but more familiar to NDT inspection people: projection $x$-ray radiography using a micro-focus $x$-ray source.

Micro-focused $x$-ray tubes for projection radiography use high-voltageaccelerated electrons focused through a high vacuum to a small spot onto a tungsten (usually) target (to be considered micro-focused, the spot diameter is less than 100 micrometers). Refer to Fig. 1. Bremstrahlung $x$-rays radiate from that spot in all directions. Their energy covers a spectrum (non-monochromatic) but peaks at about half the acceleration voltage. By intercepting the radiated 'beam' with a specimen, it is differentially absorbed more or less depending on local density, and passes through to cast an x-ray shadow. By placing a screen made with a phosphor or scintillator material down-stream, the shadow image is converted to visible light. The converted $x$-ray image can be transformed to an electronic image by coupling the scintillation screen to a camera and recording the events on video tape or as digital images in a computer. One might also use film to convert the image if real-time information or specimen motion are not issues.

Placing the screen farther away expands the image since the 'beam' diverges. This produces magnification that gives rise to $x$-ray microscopy. Clearly, there is a limit to the magnification and resolution. One limit is set by the spot size which establishes the uncertainty in the edges of the shadows. Another is the brightness of the image at the scintillator and the resolution of the scintillator screen established by the particle size in the coating. One tries the best one can to use the latest technologies to maximize sensitivity and resolution (note: this means expensive). Our workhorse imaging system is based on a $9^{\prime \prime}$ diameter demagnifying electrostaticly focused $x$-ray intensifier often seen in real-time systems for NDT or medical applications

The two $x$-ray source types are determined by the target geometry, solid 
tungsten anodes or thin tungsten films deposited (usually) on a beryllium wafer to form a transmissive window for the $x$-rays (Fig. 1). Most conventional $x$-ray tubes are the former, while the best, highest resolution devices are the latter. Since the spot size is all-important, the thin (3-10 micrometer thick) tungsten layer of the transmission-window tube will maintain the desired spot size. A thinner film reduces self-absorption to provide more flux. The state of the art for spot size is sub-micron, and concomitantly, the resolution of the $x$-ray shadow is limited to this value. A big advantage of the transmission window design is to permit the specimen to be placed close to the target window. It is not unreasonable to regularly achieve $500 \mathrm{X}$ or more true magnification. Note that whatever positions the specimen and screen have, the image will be in focus because this technique offers an infinite depth of field (i.e., no focusing over the whole magnification range).

\section{Microscopy During Solidification}

$X$-ray sources and image conversion hardware to per form the microscopy are commercially available. This hard ware is commonly found in the field of NDT inspection or for medical radiography. What is uncommon, is a furnace that permits unidirectional solidification of a metal alloy while exposing the molten material to the $x$-ray source from a distance less than $5 \mathrm{~mm}$ without imparment of the $x$-ray source. It's difficult to retain hot metal for long periods of time within millimeters of a surface one can safely touch But, this renders the greatest magnification and maximizes detectability of the smaller features. Thus, the third main part of the XTM apparatus is the special furnace. Our furnace has a water-cooled outer jacket for safety. Two opposing aluminum foil windows allow the x-rays to pass through. The long, thin, specimen is enclosed in a boron nitride crucible which is quite transparent to hard $x$-rays. The aluminum alloys to be solidified are $1 \mathrm{~mm}$ thick. The crucible is slowly driven lengthwise through the furnace along a heated tunnel. A slightly spaced pair of resistance heating elements maintain a temperature gradient along the tunnel such that the metal is liquid on one end and solid on the other. The solid-liquid interface is established near the midpoint of the furnace (where the windows and heater gap are) so one can view the interface and the cross-section of the specimen through the sides of the furnace Pulling the crucible through the furnace causes freezing of the liquid at the interface while viewing is performed normal to the growth direction. By maintaining the alloy's freezing temper ature in the middle of the furnace, the interface will appear stationary even though the specimen is being translated One would like to form the largest features possible. Generally, to do this, very low growth rates $(-5$ micrometers/sec) are used. We use a slow moving, 50 uno steplrev stepper motor tied to a fine-pitch ( 50 tpi) screw so that a nut on the screw tied to the crucible drives the specimen through the furnace. The majority of the metals we have studied to date were based on aluminum. There are many casons for choosing it

1. aluminum alloys are of commercial interest

2. aluminum has a small $x$-ray attenuation coefficient

3. aluminum alloys have a conveniently low melting point (between 600 and 660 c)

4. the density of the crystalline form is over $5 \%$ greater than the melt

5. the oxide on aluminum is strong and once formed, grows slowly in air

Continued on Page 28

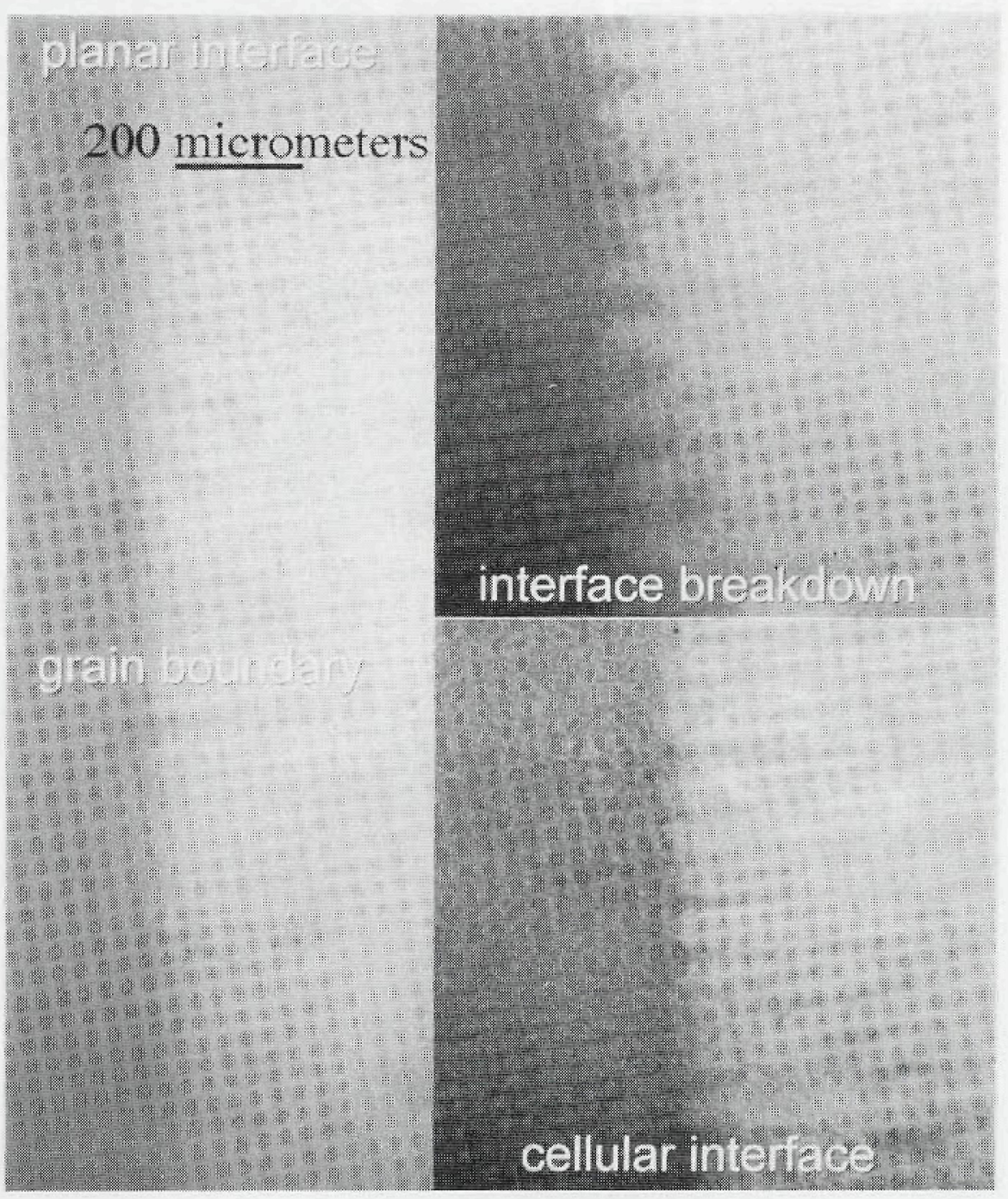

Figure 2: $\mathrm{Al} \cdot 2 \% \mathrm{Ag}$

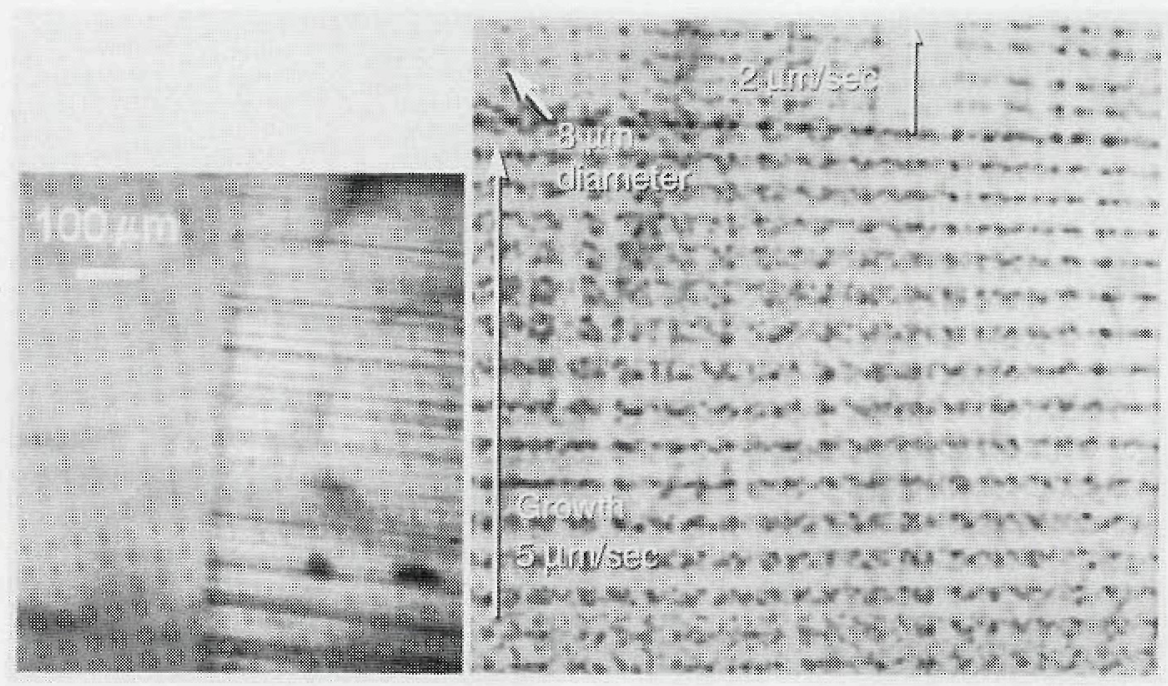

Figure 3a (left): Real-time radiomicrograph of Al-Bi monotectic alloy showing formation of arrayed droplets. Growth rate is $5 \mu \mathrm{m} / \mathrm{sec}$. Liquid is on the left and solid aluminum matrix is on the right with the s/l interface plane seen edge-on running up the center. The specimen is $1 \mathrm{~mm}$ thick and the interface is seen from a slight angle.

Figure $3 \mathrm{~b}$ (right): Post solidification radiomicrograph of three dimensional array of Bi particles in $\mathrm{Al}-\mathrm{Bi}$ monotectic. The particle spacing in the growth direction is $28.5 \mu \mathrm{m}$. 


\section{Using Projection X-ray Microscopy....}

\section{Continued from Page 27}

6. the metallurgical properties are well known and microstructures well documented

After considerable effort, it was possible to see the solid-iquid (s/l) interface of pure $\mathrm{Al}$. The $\mathrm{x}$-ray contrast was in the $2 \%$ range (depending on acceleration voltage). Our state-of-the-art imaging system could barely display the interface in real-time except when it was of sufficient thickness. Specimen thickness since that time has been kept at $1 \mathrm{~mm}$. Long exposures with film would easily show the interface if it was stationary (no motor translation). To extract the best image possible, a high resolution cooled CCD digital camera was lens-coupled to the intensifier. $X$-ray flux from a micro-focus tube is not as high as the industrial NDT $x$-ray sources offer, lest the target burn up. That is why an intensifier is needed for real-time work, and why a cooled camera is needed for its dynamic range (ours is 4,000 gray levels). If the specimen has sufficient contrast, a low-noise video camera is used to view the action from the intensifier. A VCR or frame-grabber can then be used to store the sequences of images.

The interesting observations come about when studying alloys. Selection of alloying elements involves elements with higher $Z$ than $A$ I to produce contrast and to produce interesting morphological features. We also studied metal matrix composites by adding insoluble zirconium oxide particles (high attenuation coefficient) to aluminum and some of its alloys. Often, when an alloying element is added to another. the tendency is for the solvent to reject the solute during solidification. This phenomenon may be desirable or not for the properties of the solid alloy, but it is very handy to increase the contrast at the s/ interface. Accumulation of the solute in the liquid next to the solid tends to darken it and thereby delineate the interface structure. Fig. 2 shows a sequence of images in real-time of a $2 \%$ solution of silver in aluminum. The silver accumulates in the liquid (left side) and renders it darker. This accumulation, known as the boundary layer is the focus of solidification studies. Although printing quality may make it

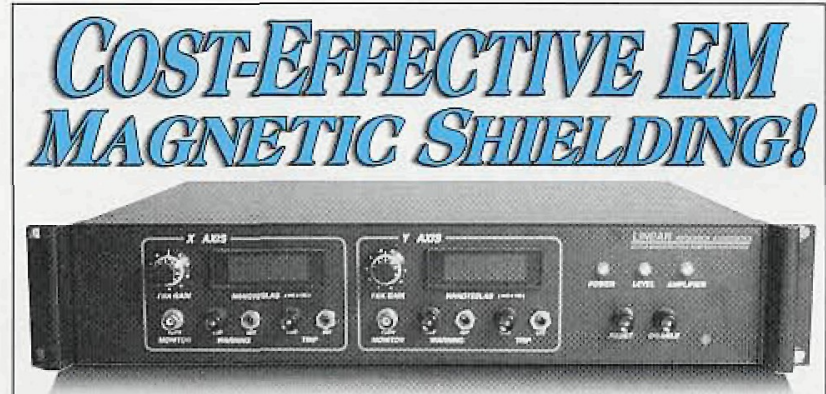

EM sites often require supplemental magnetic shielding to achieve full resolution. LINEAR RESEARCH ASSOCIATES wideband EMFC-AC electronic active-shielding systems dramatically reduce magnetic fields radiating from nearby a.c. power wiring, ground loops, transformers and related sources. EMFC-QDC extended-range models additionally suppress lowfrequency magnetic disturbances caused by trains, subways, elevators and equipment such as MRI scanners.

Linear Research Associates' EMFC equipment is the highestperformance commercially available magnetic field compensation apparatus in the world. EMFC systems feature state-of-the-art engineering $\dagger$ and are U.S.-manufactured to exacting standards. Superior performance and reliability are guaranteed!

Call or fax LRA for complete EMFC-series information. We will also gladly assist with any questions you may have regarding site survey, engineering or EMF issues.

\section{LINEAR RESEARCH ASSOCIATES}

5244 Perry City Road • Trumansburg, NY 14886

Phone (607) 387-3411 • Fax (607) 387-7806

info@linres.com - http://www.linres.com

t† U.S. Patents $5,465,012 ; 5,469,058$ difficult to see, the boundary layer is found in this figure and is visibly about 75 micrometers deep, seen as a slightly darker band against the solid. As far as we know, this is the first instance of the real-time observation of this layer. Using the $X T M$, we hope to perform quantitative analysis on the composition, concentration and extent of this layer. This sequence also shows the importance of the microstructure formed at the sil interface

The left image in the sequence shows a planar interface growing at a rate of $1 \mu \mathrm{m} / \mathrm{sec}$. While boring to look at, this interface type is important to maintain during growth of single crystals such as silicon used to make elecironic devices. Of significance is the resulting uniformity of the solute distribution throughout the crystal. The grain boundary shows that this is in fact two crystals side-by-side. This would be a major imperfection if a single crystal was desired. The second and third parts of the figure show the transition to $(1.5 \mu \mathrm{m} / \mathrm{sec}$ rate $)$ and steady-state growth of cells at $2 \mathrm{~mm} / \mathrm{sec}$. Cells were induced by increasing the translation rate. Cells are fingers of solid that form due to the solute build-up which alters the freezing temperatures over the scale of the cell diameter. The segregation of the solute into long strips (between the cells) leads to sharp variations in the composition within the solidified casting. This alters many of the properties of the material and needs to be managed in serviceable alloys. Features such as these or the related dendrite structure (not shown here) are called interfacial instabilities and either need to be suppressed, as for crystal growth, or controlled when formed in castings.

A large variety of microstructures form in the diversity of metal alloys. Many people are unaware of the patterns that particles of one material create in another. An example of such a distribution pattern, shown in Fig. $3 \mathrm{~b}$, is clearly rendered by the XTM. Here we see one morphology that is the product of a monotectic reaction: a liquid solution of bismuth in aluminum separates (unmixes) into droplets (about 4 micrometers radius) of bismuth surrounded by a matrix of solid aluminum during solidification. Conventional metallographic sectioning and polishing would reveal a regular array of bismuth particles but could not show that indeed the array is of an arrangement of particles that has periodicity in all 3 dimensions. Fig. 3 a shows the real-time radiomicrograph during solidification. In this case, the droplets form at (i.e., in) the sill interface. while our experiments with other monotectic alloys have shown the droplets can form in the liquid anead of the sil interface (Al-|n) or in the form of continuous fibers (AI-Po and $\mathrm{Al}-\mathrm{Bi}$ ). Before the XTM, we could only speculate how the 3 dimensional array developed. The XTM showed that the flat s/l interface develops a periodic array of droplets all over its 'face' and that this array forms periodically, in time, as the interface advances by solidification. The distance between rows of particles in the growth direction is about 28 micrometers. By looking at the specimen from different angles in the XTM. one finds the spacing between the particles is about the same order of magnitude in the other directions as well. The features in Fig. 3 have an inherently high $x$-ray contrast due to their high density. This fact, coupled with the inherent resolution of the XTM makes such small particles easy to see this way. In comparison. had the particles been made with $\mathrm{SiC}$, a reinforcement material for aluminum. even 100 micrometer particles would difficult to resolve. This illustrates that sometimes desirable features might not be visible with the XTM and alloy selection is an important component of these investigations.

For more information on this project, and journal references. see the web pages at: http:/www.ssl.msfc.nasa.gov/ssl/msad/xray/

\section{Acknowledgments}

The support of NASA's Office of Life and Microgravity Sciences and Applications for the last 4 years and of the State of Alabama before that. is gratefully acknowledged. I wish to especially recognize some people for their contributions to this work: Dr. Franz Rosenberger of the CMMR at UAH, for guidance from the beginning of this program; Dr. Peter Curreri of NASA at MSFC. for his partnership in this project; and the handful of people at MSFC and UAH that have and still are contributing to this project.

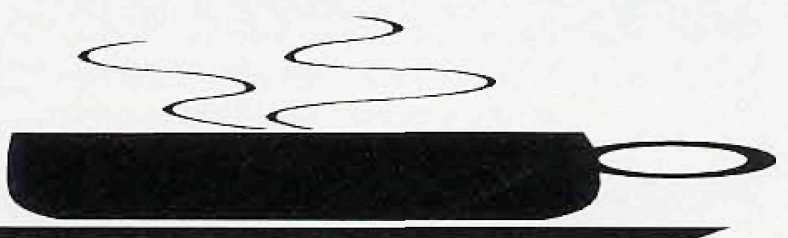




\section{X-Ray Analysis Made

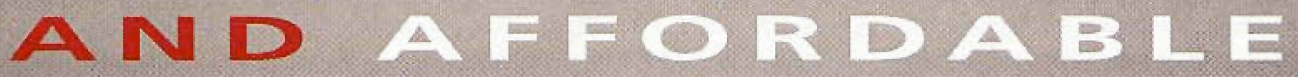

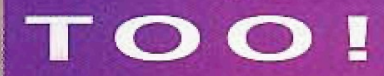

Truly an investment, the new PERSONAL EDS ${ }^{\otimes}$ delivers the best price/periormance ratio available today. Simple to use yet quite sophisticated, the new light element $X$-ray analysis system features a Gresham Sirius detector producing better than $130 \mathrm{eV}$ resolution with the lowest background counts available in the industry today. An ultrathin window allows superior light element detection.

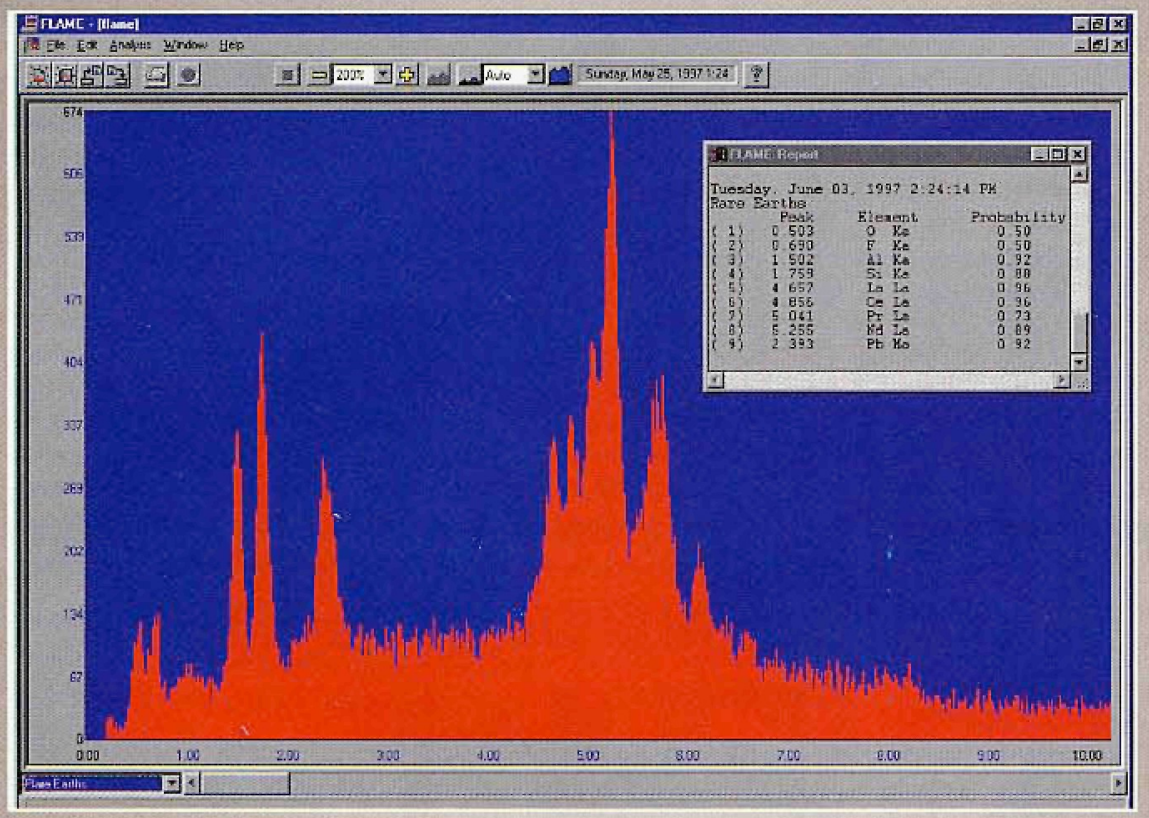

Designed with operational simplicity in mind, this PC-based detector uses Fuzzy Logic and an Artificial Intelligence database that allow the user to concentrate on sample acquisition and quantitative analysis rather than procedures. Results are obtained quickly, efficiently and in real-time. Most importanily, the hardware and software of the PERSONAL EDS ${ }^{\circledR}$ have been fully integrated by skilled professionals at RJ Lee Instruments, manufacturer of the PERSONAL SEM ${ }^{\circledR}$, and backed by a worldwide technical support team.

\section{Retroittiable to most models of SEMs or available on the PERSONAL SEMW I Supported by the application experts who created the PERSOWAL SEM Available for under $\$ 45 K$ (1/}

